\title{
DISAL Glycosyl Donors for the Synthesis of a Linear Hexasaccharide under Mild Conditions
}

\author{
Lars Petersen ${ }^{\dagger}$, Jane B. Laursen ${ }^{\dagger}$, Kim Larsen $^{\$}$, M. Saddik Motawia $\$$, \\ and Knud J. Jensen* \\ Department of Chemistry, The Royal Veterinary and Agricultural University, 40 \\ Thorvaldsensvej, DK-1871 Frederiksberg C, Denmark. \\ 'Department of Chemistry, Technical University of Denmark, Building 201, Kemitorvet, \\ DK-2800 Kgs. Lyngby, Denmark.

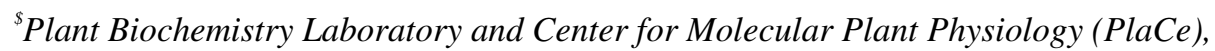 \\ Department of Plant Biology, The Royal Veterinary and Agricultural University, 40 \\ Thorvaldsensvej, DK-1871 Frederiksberg C, Denmark. \\ $k j j @ k v l . d k$
}

SUPPORTING INFORMATION

EXPERIMENTAL PROCEDURES INCLUDING SPECTROSCOPIC AND ANALYTICAL DATA

General Procedures: Molecular sieves (4A) were activated under high vacuum at $150^{\circ} \mathrm{C}$ for 24h. $\mathrm{CH}_{3} \mathrm{NO}_{2}$ was purified by vacuum distillation to remove the water/ $\mathrm{CH}_{3} \mathrm{NO}_{2}$ azeotrop, followed by drying with $\mathrm{CaSO}_{4}$, and stored in dark bottles over $4 \AA$ molecular sieves. Water contents $(<20 \mathrm{ppm})$ were measured by Karl-Fischer titration. All other solvents were distilled and/or stored over $3 \AA$ or $4 \AA$ molecular sieves as appropriate. ${ }^{1} \mathrm{H}$ NMR spectra were recorded on either a Varian Mercury 300 operating at $300.06 \mathrm{MHz}$ equipped with a 4-nuclei probe or a Varian Unity Inova 500 operating at 499.87 MHz equipped with a z- (single axis) PFG inverse detection C-H-P probe. ${ }^{13} \mathrm{C}-\mathrm{NMR}$ were recorded on a Varian Mercury 300 operating at $75.46 \mathrm{MHz}$ or a Varian Unity Inova 500 operating at 125 MHz. Chemical shift $(\delta)$ values are in ppm, coupling constants $(J)$ are in Hz. All assignments were supported by 2D homonuclear chemical-shift correlation spectroscopy (gCOSY) and in some cases heteronuclear single quantum correlated spectroscopy (gHSQC) experiments. Vacuum liquid chromatography (VLC) was carried on Merck Silica Gel 60H. HPLC analyses were conducted with a Waters system (600 control unit, 996 photodiode array (PDA) detector, 717 Plus autosampler, Millenium 32 control software) on a Waters Nova-Pak $\mathrm{C}_{18}$ column $(3.9 \times 50 \mathrm{~mm}$ cartridge; $4 \mu \mathrm{m}$ particle size) or a Waters Symmetry $300 \mathrm{C}_{4}$ column $(3.9 \times 150 \mathrm{~mm} ; 5 \mu \mathrm{m}$ particle size $)$ using a linear gradient of $0.1 \%$ aq. TFA (A) and $0.1 \%$ TFA in $\mathrm{CH}_{3} \mathrm{CN}(\mathrm{B}): 0$ min: $0 \% \mathrm{~B}, 2$ min: $0 \% \mathrm{~B}, 5$ min: $50 \% \mathrm{~B}, 12 \mathrm{~min}$ : $95 \%$ B, 13 min: $95 \%$ B, $13.5 \mathrm{~min}: 0 \%$ B, $20 \mathrm{~min}: 0 \% \mathrm{~B}$. Monitoring was from 200 to $400 \mathrm{~nm}$, integrations were performed at 215 and $265 \mathrm{~nm}$, and individual peaks were analyzed by their UVspectra. The purity of compounds was determined from integrations at $215 \mathrm{~nm}$. Preparative HPLC purifications were conducted with the above system on three consecutive Prep Nova-Pak $\mathrm{C}_{18}$ columns $\left(40 \times 100 \mathrm{~mm} ; 6 \mu \mathrm{m}\right.$ particle size) or a Waters PrepPak $\mathrm{C}_{4}$ cartridge (Delta-Pak ${ }^{\mathrm{TM}}, 25 \times 100 \mathrm{~mm} ; 15 \mu \mathrm{m}$ particle size). MS analyses were performed on a Micromass LCT mass spectrometer. 
Synthesis of donors 4 and 5: DISAL glycosyl donors were synthesized as previously described ${ }^{\mathrm{i}}$ with small changes in the work-up procedure. The crude reaction mixture was transferred to a packed VLC column of ovendried silica gel $\left(110^{\circ} \mathrm{C}\right.$ for several days). Elution was performed using EtOAc and toluene (1:15) stored over $4 \AA$ molecular sieves. This procedure raised isolated yields of disaccharide donors from approx. 50-60\% to almost $90 \%$ as discussed in the main text.

\section{Data for 2-carboxymethyl-4,6-dinitrophenyl 2,3,6-tri-O-benzyl-4-(2,3,4,6-tetra-O-benzyl- $\alpha$-D- glucopyranosyl)- $\alpha$-D-glucopyranoside $4 \alpha$}

Colorless foam from $\mathrm{CH}_{2} \mathrm{Cl}_{2}$ stored at $-10^{\circ} \mathrm{C} .{ }^{1} \mathrm{H}$ NMR $\left(500 \mathrm{MHz}, \mathrm{CDCl}_{3}\right): \delta 8.68(\mathrm{~d}, 1 \mathrm{H}, \mathrm{J}=3.0), 8.57$ $(\mathrm{d}, 1 \mathrm{H}, \mathrm{J}=3.0), 7.32-6.93(\mathrm{~m}, 35 \mathrm{H}), 5.63(\mathrm{~d}, 1 \mathrm{H}, \mathrm{J}=3.8 ; \mathrm{H}-1$ ' $\alpha$; HSQC showed strong coupling to 97.34 in $\left.{ }^{13} \mathrm{C}\right), 5.41\left(\mathrm{~d}, 1 \mathrm{H}, \mathrm{J}=3.0\right.$; $\mathrm{H}-1 \alpha$; HSQC shows strong coupling to 104.14 in $\left.{ }^{13} \mathrm{C}\right), 4.96-4.28(\mathrm{~m}$, $14 \mathrm{H}$ ), $4.21(\mathrm{dd}, 1 \mathrm{H}, \mathrm{J}=8.1$ and 9.0), $4.14(\mathrm{dd}, 1 \mathrm{H}, \mathrm{J}=8.1$ and 9.4), $4.10($ br. $\mathrm{d}, 1 \mathrm{H}, \mathrm{J} \sim 9.4$ ), 3.97 (dd, $1 \mathrm{H}, \mathrm{J}=9.4$ ), $3.92(\mathrm{dd}, 1 \mathrm{H}, \mathrm{J}=3.0$ and 11.1), 3.85 (s, 3H), 3.82 (br. d, $1 \mathrm{H}, \mathrm{J} \sim 9.8), 3.71-3.63(\mathrm{~m}, 3 \mathrm{H})$, $3.59(\mathrm{dd}, 1 \mathrm{H}, \mathrm{J}=3.4$ and 10.7$), 3.54(\mathrm{dd}, 1 \mathrm{H}, \mathrm{J}=3.8$ and 9.8$), 3.48(\mathrm{dd}, 1 \mathrm{H}, \mathrm{J}=1.7$ and 10.7$) .{ }^{13} \mathbf{C}$ NMR $\left(75 \mathrm{MHz}, \mathrm{CDCl}_{3}\right): \delta 163.87,154.43,144.98,141.50,138.95,138.90,138.68,138.37,138.19,138.17$, 137.08, 129.75, 129.25, 128.6-127.5 (m), 127.47, 127.12, 125.52, 123.58, 104.14, 97.34, 82.16, 81.23, 80.92, 79.86, 77.91, 75.75, 75.19, 74.87, 74.47, 73.90, 73.71, 73.47, 73.40, 72.64, 71.39, 68.61, 68.56, 53.59. ESI-MS: $\mathrm{m} / z$ calcd. for $\mathrm{C}_{69} \mathrm{H}_{72} \mathrm{~N}_{3} \mathrm{O}_{17}\left(\mathrm{M}+\mathrm{NH}_{4}\right)^{+} 1214.49$, found $1214.61 ; \mathrm{m} / z$ calcd. for $\mathrm{C}_{69} \mathrm{H}_{68} \mathrm{~N}_{2} \mathrm{NaO}_{17}(\mathrm{M}+\mathrm{Na})^{+} \mathrm{m} / \mathrm{z} 1219.44$, found 1219.55

Data for 2-carboxymethyl-4,6-dinitrophenyl 2,3,-di-O-benzyl-6-O-trityl-4-(2,3,4,6-tetra-O-benzyl- $\alpha-D$ glucopyranosyl)- $\alpha$-D-glucopyranoside $5 \alpha{ }^{1} \mathbf{H}$ NMR $\left(500 \mathrm{MHz}, \mathrm{CDCl}_{3}\right.$, see also Figure 1): $8.67(\mathrm{~d}, 1 \mathrm{H}$, $\mathrm{J}=6$ ), $8.57(\mathrm{~d}, 1 \mathrm{H}, \mathrm{J}=6), 7.48-7.06(41 \mathrm{H}), 6.95$ (br. d, $2 \mathrm{H}, \mathrm{J}=7), 6.90$ (br. d, $2 \mathrm{H}, \mathrm{J}=7), 5.62(\mathrm{~d}, 1 \mathrm{H}, \mathrm{J}$ $=3.84), 5.43(\mathrm{~d}, 1 \mathrm{H}, \mathrm{J}=3.41), 5.08-4.28(12 \mathrm{H}), 4.21(\mathrm{~m}, 2 \mathrm{H}), 4.11(\mathrm{~m}, 1 \mathrm{H}), 4.00(1 \mathrm{H}, \mathrm{J}=11.1, \mathrm{~J}=3.0)$, $3.97(\mathrm{t}, 1 \mathrm{H}, \mathrm{J}=9.4), 3.94(\mathrm{dd}, 1 \mathrm{H}, \mathrm{J}=8.1, \mathrm{~J}=2.1), 3.85\left(3 \mathrm{H}, \mathrm{CH}_{3}\right), 3.79(1 \mathrm{H}), 3.70(\mathrm{dd}, 1 \mathrm{H}, \mathrm{J}=8.5, \mathrm{~J}=$ 3.4), $3.64(\mathrm{dd}, 1 \mathrm{H}, \mathrm{J}=9.81, \mathrm{~J}=3.4), 3.49(\mathrm{dd}, 1 \mathrm{H}, \mathrm{J}=10.2, \mathrm{~J}=1.3), 3.13(\mathrm{dd}, 1 \mathrm{H}, \mathrm{J}=10.2, \mathrm{~J}=3.8) .{ }^{13} \mathrm{C}$ NMR $\left(125 \mathrm{MHz}, \mathrm{CDCl}_{3}\right): \delta 164.38,154.93,145.47,144.65,141.96,139.63,139.38 ., 138.96,138.84$, 138.58, 137.67, 130.3-127 (21 C), 124.07, 104.57, 97.20, 87.04, 82.68, 81.34, 81.26, 80.94, 78.66, 76.52, 75.68, 75.29, 74.92, 74.32, 73.88, 73.76, 72.06, 69.09, 63.09, 54.07. ESI-MS: $\mathrm{m} / z$ calcd. for $\mathrm{C}_{81} \mathrm{H}_{76} \mathrm{~N}_{2} \mathrm{NaO}_{17}(\mathrm{M}+\mathrm{Na})^{+}$1371.50, found 1371.38.

Optimization of glycosylation conditions (Table 1): Disaccharide glycosyl donor 4 or 5 (typically 0.01 $\mathrm{mmol}$ ) and promoter(s) were dissolved in solvent $(600 \mu \mathrm{L})$, and cyclohexanol (stored over $4 \AA ̊$ molecular sieves) was added followed by mixing. After shaking for the indicated period on an Eppendorf Thermomixer 5436 (combined heater/shaker), a sample $(40 \mu \mathrm{L})$ was diluted in acetonitrile $(0.6 \mathrm{~mL})$ and analyzed by analytical HPLC. Reported yields were based on integrated areas of products formed (215 $\mathrm{nm})$. From a series of test reactions, the product was purified by preparative HPLC and characterized.

Cyclohexyl 2,3,6-tri-O-benzyl-4-(2,3,4,6-tetra-O-benzyl- $\alpha$-D-glucopyranosyl)- $\alpha$-D-glucopyranoside $6 \alpha$ ${ }^{1}$ H NMR (500 MHz, $\left.\mathrm{CDCl}_{3}\right): \delta 7.31-7.11(\mathrm{~m}, 35 \mathrm{H}), 5.72(\mathrm{~d}, 1 \mathrm{H}, J=3.4), 5.07(\mathrm{~d}, 1 \mathrm{H}, J=11.5), 4.98$ $(\mathrm{d}, 1 \mathrm{H}, J=3.4), 4.91(\mathrm{~d}, 1 \mathrm{H}, J=10.7), 4.84-4.78(\mathrm{~m}, 3 \mathrm{H}), 4.67-4.48(\mathrm{~m}, 7 \mathrm{H}), 4.45(\mathrm{~d}, 1 \mathrm{H}, J=10.7)$, $4.31(\mathrm{~d}, 1 \mathrm{H}, J=12.4), 4.12(\mathrm{dd}, 1 \mathrm{H}, J=8.5$ and 9.0$), 4.05(\mathrm{dd}, 1 \mathrm{H}, J=8.5$ and 9.8$), 4.01$ (ddd, $1 \mathrm{H}, J \approx 2$ and 9.8), $3.94(\mathrm{dd}, 1 \mathrm{H}, J=9.4), 3.87(\mathrm{dd}, 1 \mathrm{H}, J=3.8$ and 10.7), 3.77 (ddd, $1 \mathrm{H}, J \approx 2$ and 9.8), 3.69-3.64 $(\mathrm{m}, 2 \mathrm{H}), 3.62(\mathrm{dd}, 1 \mathrm{H}, J=3.8$ and 9.4$), 3.60-3.53(\mathrm{~m}, 1 \mathrm{H}), 3.53-3.49(\mathrm{~m}, 2 \mathrm{H}), 3.43(\mathrm{dd}, 1 \mathrm{H}, J=1.7$ and 10.7), 1.96-1.89 (m, 2H), 1.84-1.75 (m, 2H), 1.60-1.20 (m, 6H). $\left.{ }^{13} \mathbf{C ~ N M R ~ ( 7 5 ~ M H z , ~} \mathrm{CDCl}_{3}, 30 \mathrm{mg}\right): \delta$ 139.39, 139.07, 138.76, 138.57, 138.36, 138.24, 138.20, 128.6-126.93 (m), 96.81, 94.68, 82.23, 82.06, 80.60, 79.78, 77.98, 75.96, 75.73, 75.14, 74.34, 73.69, 73.34, 73.09, 72.89, 71.20, 69.84, 69.48, 68.54, 33.70, 31.86, 25.89, 24.75, 24.49. RP-HPLC $\left(\mathrm{R}_{\mathrm{t}} 21.09 \mathrm{~min}, \mathrm{C}_{18}\right.$ column, $\left.215 \mathrm{~nm}\right)>99 \%$. ESI-MS: $\mathrm{m} / z$ 
calcd. for $\mathrm{C}_{67} \mathrm{H}_{78} \mathrm{NO}_{11}\left(\mathrm{M}+\mathrm{NH}_{4}\right)^{+} 1072.56$, found $1072.55(100 \%) ; \mathrm{m} / z$ calcd. for $\mathrm{C}_{67} \mathrm{H}_{74} \mathrm{NaO}_{11}(\mathrm{M}+\mathrm{Na})^{+}$ $\mathrm{m} / \mathrm{z}$ 1077.51, found $1077.52(35 \%)$.

Cyclohexyl 2,3,6-tri-O-benzyl-4-(2,3,4,6-tetra-O-benzyl- $\alpha$-D-glucopyranosyl)- $\beta$-D-glucopyranoside $6 \beta$ ${ }^{1}$ H NMR $\left(500 \mathrm{MHz}, \mathrm{CDCl}_{3}\right): \delta 7.32-7.11(\mathrm{~m}, 35 \mathrm{H}), 5.68(\mathrm{~d}, 1 \mathrm{H}, \mathrm{J}=3.7), 4.98-4.93(\mathrm{~m}, 2 \mathrm{H}), 4.87(\mathrm{~d}$, $1 \mathrm{H}, \mathrm{J}=11.5), 4.79(\mathrm{~d}, 1 \mathrm{H}, \mathrm{J} \sim 8), 4.77(\mathrm{~d}, 1 \mathrm{H}, \mathrm{J} \sim 7), 4.76(\mathrm{~d}, 1 \mathrm{H}, \mathrm{J}=11.5), 4.61-4.45(\mathrm{~m}, 8 \mathrm{H}), 4.36(\mathrm{~d}$, $1 \mathrm{H}, \mathrm{J}=11.5), 4.03(\mathrm{dd}, 1 \mathrm{H}, \mathrm{J}=8.6$ and 9.3), $3.90(\mathrm{dd}, 1 \mathrm{H}, \mathrm{J}=9.2$ and 9.5), 3.81-3.69 (m, 5H), 3.66-3.46 $(\mathrm{m}, 6 \mathrm{H}), 2.04-1.20(\mathrm{~m}, 10 \mathrm{H}) .{ }^{13} \mathbf{C}$ NMR $\left(75 \mathrm{MHz}, \mathrm{CDCl}_{3}\right.$; selected data): $\delta 139.62,139.51,139.21$, 139.19, 139.10, 138.72, 102.66, 97.42, 34.55, 32.80, 30.39, 26.36, 24.72. RP-HPLC $\left(\mathrm{R}_{\mathrm{t}} 21.32 \mathrm{~min}, \mathrm{C}_{18}\right.$ column, $215 \mathrm{~nm}):>99 \%$. ESI-MS: $\mathrm{m} / z$ calcd. for $\mathrm{C}_{67} \mathrm{H}_{78} \mathrm{NO}_{11}\left(\mathrm{M}+\mathrm{NH}_{4}\right)^{+} 1072.56$, found 1072.55 $(100 \%) ; m / z$ calcd. for $\mathrm{C}_{67} \mathrm{H}_{74} \mathrm{NaO}_{11}(\mathrm{M}+\mathrm{Na})^{+} \mathrm{m} / \mathrm{z} 1077.51$, found $1077.52(45 \%)$.

General procedure for glycosylation reactions A $5 \mathrm{~mL}$ cryotube was charged with glycosyl donor $(0.075 \mathrm{mmol})$, glycosyl acceptor $(0.050 \mathrm{mmol})$, activators and additives if required, a magnet, and crushed $4 \AA$ molecular sieves. The tubes were mounted with a suitable rubber septum, which was then pierced with a needle. The closed tube was then evacuated under high vacuum; Ar was let in followed by re-evacuation. This cycle was repeated twice and then left to dry for $1-2 \mathrm{~h}$. Ar was let in, the needle was removed, and finally dry solvent $(1 \mathrm{~mL})$ was added by means of a syringe. The reaction mixture was then stirred magnetically while heating by means of an oil bath if required. Work-up consisted of filtration $(0.45 \mu \mathrm{m})$ and purification by preparative HPLC followed by evaporation of appropriate fractions. In cases where the reaction mixture was poorly filterable due to the crushed molecular sieves and additives, it was eluted through a short plug of silica gel using a $\mathrm{CH}_{2} \mathrm{Cl}_{2}-\mathrm{MeOH}$ mixture. The residue upon concentration was then purified by preparative HPLC.

Data for thiophenyl 2,3,6-tri-O-benzyl-4-(2,3,4-tri-O-benzyl-6-[2,3,6-tri-O-benzyl-4-(2,3,4,6-tetra-Obenzyl- $\alpha$-D-glucopyranosyl)- $\alpha$-D-glucopyranosyl]- $\alpha$-D-glucopyranosyl)- $\beta$-D-glucopyranoside

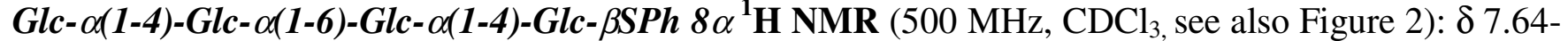
$7.59(\mathrm{~m}, 2 \mathrm{H}), 7.38-7.08(\mathrm{~m}, 68 \mathrm{H}), 5.73(\mathrm{~d}, 1 \mathrm{H}, \mathrm{J}=3.4 ; \mathrm{H}-1 \alpha), 5.56(\mathrm{~d}, 1 \mathrm{H}, \mathrm{J}=3.4 ; \mathrm{H}-1 \alpha), 5.17(\mathrm{~d}, 1 \mathrm{H}, \mathrm{J}$ $=3.8 ; \mathrm{H}-1 \alpha$; all three $\alpha$-anomeric signals shows strong coupling to $\sim 97 \mathrm{ppm}$ in $\left.{ }^{13} \mathrm{C}\right), 5.05-3.20(\mathrm{~m}$, 54H) ${ }^{13} \mathbf{C}$ NMR $\left(75 \mathrm{MHz}, \mathrm{CDCl}_{3}\right): \delta 139.25,139.08,139.05,138.90,138.85,138.82,138.73,138.60$, 138.43, 138.31, 138.26, 138.24, 138.09, 134.11, 133.88, 132.26, 132.11, 139.14, 128.7-126.5 (m), 97.05, 96.98, 96.88, 87.48, 87.10, 82.31, 82.13, 81.87, 81.09, 80.38, 79.73, 78.95, 77.95, 77.47, 75.70, 75.60, $75.47,75.28,75.14,75.08,74.35,74.19,73.71,73.68,73.52,73.47,73.21,72.66,72.50,72.19,71.80$, 71.21, 70.11, 69.35, 69.27, 68.53, 65.18. ESI-MS: $\mathrm{m} / \mathrm{z}$ calcd. for $\mathrm{C}_{121} \mathrm{H}_{124} \mathrm{KO}_{20} \mathrm{~S}(\mathrm{M}+\mathrm{K})^{+} \mathrm{m} / \mathrm{z}$ 1967.80, found $1967.81(65 \%) ; \mathrm{m} / z$ calcd. for $\mathrm{C}_{121} \mathrm{H}_{124} \mathrm{NaO}_{20} \mathrm{~S}(\mathrm{M}+\mathrm{Na})^{+} 1951.83$, found $1951.69(50 \%)$.

Data for thiophenyl 2,3,6-tri-O-benzyl-4-(2,3,4-tri-O-benzyl-6-[2,3,6-tri-O-benzyl-4-(2,3,4,6-tetra-Obenzyl- $\alpha$-D-glucopyranosyl)- $\beta$-D-glucopyranosyl]- $\alpha$-D-glucopyranosyl)- $\beta$-D-glucopyranoside $\quad$ Glc$\alpha(\mathbf{1 - 4})-\boldsymbol{G l c}$ - $\beta(\mathbf{1 - 6})-\boldsymbol{G l c}$ - $\alpha(\mathbf{1 - 4})-\boldsymbol{G l c}$ - $\beta$ SPh $8 \boldsymbol{\beta}^{\mathbf{1}} \mathbf{H} \mathbf{N M R}\left(500 \mathrm{MHz}, \mathrm{CDCl}_{3}\right.$; selected data): $\delta 5.68$ (br. d, $2 \mathrm{H}, \mathrm{J} \sim 3.4 ; 2 \times \mathrm{H}-1 \alpha$, HSQC showed strong coupling to 97.4 in $\left.{ }^{13} \mathrm{C}\right) .{ }^{13} \mathbf{C ~ N M R}\left(125 \mathrm{MHz}, \mathrm{CDCl}_{3}\right.$; selected data): $\delta 104.41,97.44,88.10,87.48,85.61,82.74,82.70,82.52,81.77,80.13,79.78,79.37$, 78.71, 78.50, 76.16, 76.07, 75.87, 75.65, 75.55, 75.52, 75.50, 74.86, 74.51, 74.19, 74.13, 73.98, 73.92, 73.30, 73.17, 71.73, 71.47, 70.07, 69.78, 69.03. ESI-MS: $\mathrm{m} / z$ calcd. for $\mathrm{C}_{121} \mathrm{H}_{124} \mathrm{KO}_{20} \mathrm{~S}(\mathrm{M}+\mathrm{K})^{+} \mathrm{m} / \mathrm{z}$ 1967.80, found $1967.81(65 \%) ; \mathrm{m} / z$ calcd. for $\mathrm{C}_{121} \mathrm{H}_{124} \mathrm{NaO}_{20} \mathrm{~S}(\mathrm{M}+\mathrm{Na})^{+} 1951.83$, found $1951.69(50 \%)$.

Deprotection of tritylated tetrasaccharide, thiophenyl 2,3,6-tri-O-benzyl-4-(2,3,4-tri-O-benzyl-6-[2,3,6tri-O-benzyl-4-(2,3,4-tri-O-benzyl-6-O-trityl- $\alpha$-D-glucopyranosyl)- $\alpha / \beta$-D-glucopyranosyl]- $\alpha-D$ -

glucopyranosyl)- $\beta$-D-glucopyranoside 10 The crude product from a glycosylation reaction using tritylated donor 5 and acceptor 7 after elution through a plug of silica gel using $\mathrm{CH}_{2} \mathrm{Cl}_{2}-\mathrm{MeOH}(98: 2)$ and concentration was dissolved in a mixture of glacial acetic acid, water and acetonitrile (approx. 
90:7:3) was heated at $60^{\circ} \mathrm{C}$ by means of an oil bath for $3 \mathrm{~h}$. A sample was withdrawn from the reaction mixture and analyzed by analytical HPLC, which showed no presence of trityl-protected tetrasaccharide. The reaction mixture was concentrated and once reconcentrated from toluene. The residue was dissolved in acetonitrile, filtered, and purified by preparative HPLC using a $\mathrm{C}_{4}$-column. Concentration of appropriate fractions yielded $34.5 \mathrm{mg}$ of deprotected material, which amounted to $38 \%$ yield for the glycosylation step and deprotection (2 steps). ${ }^{1} \mathbf{H}$ NMR (500 MHz, $\mathrm{CDCl}_{3}$, selected data): $\delta 5.69(\mathrm{~d}, \mathrm{~J}=$ 3.4; minor isomer), 5.66 (d, $\mathrm{J}=3.8$; major isomer), 5.65 (d, $\mathrm{J}=3.8$; minor isomer), $5.56(\mathrm{~d}, \mathrm{~J}=3.4$; major isomer), $5.14(\mathrm{~d}, \mathrm{~J}=3.4$; major isomer). Ratio major-minor $\sim 1.4: 1$. As three anomeric protons with $\alpha$-configuration were present in the major isomer, it was assigned an anomeric configuration of $\alpha, \alpha, \alpha, \beta \mathrm{SPh}$ and the minor isomer was assigned to $\alpha, \beta, \alpha, \beta \mathrm{SPh}$.

Hexasaccharide thiophenyl 2,3,6-tri-O-benzyl-4-(2,3,4-tri-O-benzyl-6-[2,3,6-tri-O-benzyl-4-(2,3,4-triO-benzyl-6-[2,3,6-tri-O-benzyl-4-(2,3,4,6-tetra-O-benzyl- $\alpha$-D-glucopyranosyl)- $\alpha \beta$-D-glucopyranosyl]$\alpha$-D-glucopyranosyl)- $\alpha \beta$-D-glucopyranosyl]- $\alpha$-D-glucopyranosyl)- $\beta$-D-glucopyranoside 11.

Tetrasaccharide acceptor 10 (19 mg, $10 \mu \mathrm{mol}, 1$ equiv), disaccharide DISAL donor 4 (19 mg, 1.5 equiv), $\mathrm{LiClO}_{4}\left(7 \mathrm{mg}, 6\right.$ equiv), $\mathrm{Li}_{2} \mathrm{CO}_{3}(5 \mathrm{mg}, 6$ equiv.) and crushed $4 \AA$ molecular sieves $(20 \mathrm{mg})$ were placed in plastic tube with magnet and sealed with a septum. The reagents were dried in high-vacuum for min. 1h, After Ar inlet, 1,2-dichloroethane $\left(250 \mu \mathrm{L}\right.$, kept over $4 \AA \mathrm{MS}$, dry, $\left.<5 \mathrm{ppm} \mathrm{H}_{2} \mathrm{O}\right)$ was added and the reaction was stirred for $0.5 \mathrm{~h}$ at room temperature. The temperature was raised to $35^{\circ} \mathrm{C}$ and stirring was continued for $1 \mathrm{~d}$. Additionally, 1.5 equiv of disaccharide DISAL donor $4(0.075 \mathrm{mmol})$ was added and the stirring was continued for 2 days. Additionally, 6 equiv of $\mathrm{LiClO}_{4}$ was added and the stirring was continued for $1 \mathrm{~d}$. Additionally, 1.5 equiv of disaccharide DISAL donor 4 (0.075 mmol) was added and the stirring was continued for 2 days. The reaction mixture was filtered and loaded onto a preparative $\mathrm{C}_{4}$ HPLC column and eluted with a gradient of $\mathrm{CH}_{3} \mathrm{CN}$ and water from $10 \%$ to $95 \% \mathrm{CH}_{3} \mathrm{CN}$ over 70 min. Hereby, hexasaccharide $11(\alpha / \beta \sim 3: 2)$ was isolated as a colorless oil $(11 \mathrm{mg}, 38 \%)$ and the acceptor $(8$ $\mathrm{mg}, 50 \%)$ was recovered. The different diastereomeric hexasaccharide products were not perfectly separable on an analytical $\mathrm{C}_{4} \mathrm{HPLC}$ column ( $\left.\mathrm{R}_{\mathrm{t}} 25.07 \mathrm{~min}\right)$ but NMR analysis and an estimate based on HPLC indicated an $\alpha / \beta$ ratio of the last formed 1,6-linkage of $\sim 3: 2 .{ }^{1} \mathbf{H} \mathbf{~ N M R}\left(500 \mathrm{MHz}, \mathrm{CDCl}_{3}\right.$, see also Figure 3): $\delta$ 7.6-7.55 (m, 2H), 7.35-7.02 (m, 98H), $5.78(\mathrm{~d}, 1 \mathrm{H}$, minor isomer), 5.72-5.62 (br. d, 1H, major isomer), $5.58(\mathrm{~d}, 1 \mathrm{H}), 5.53(\mathrm{~d}, 1 \mathrm{H}), 5.51(\mathrm{~d}, 1 \mathrm{H}), 5.16-5.12$ (br. d, 1H, major isomer), $5.07(\mathrm{~d}, 1 \mathrm{H}$, minor isomer), 5.0-4.2 (m, 35H), 4.14-3.16 (m, 41H). ESI-MS: $m / z$ calcd. for $\mathrm{C}_{175} \mathrm{H}_{180} \mathrm{NaO}_{30} \mathrm{~S}(\mathrm{M}+\mathrm{Na})^{+}$ 2816.2, found: 2816.9 . 


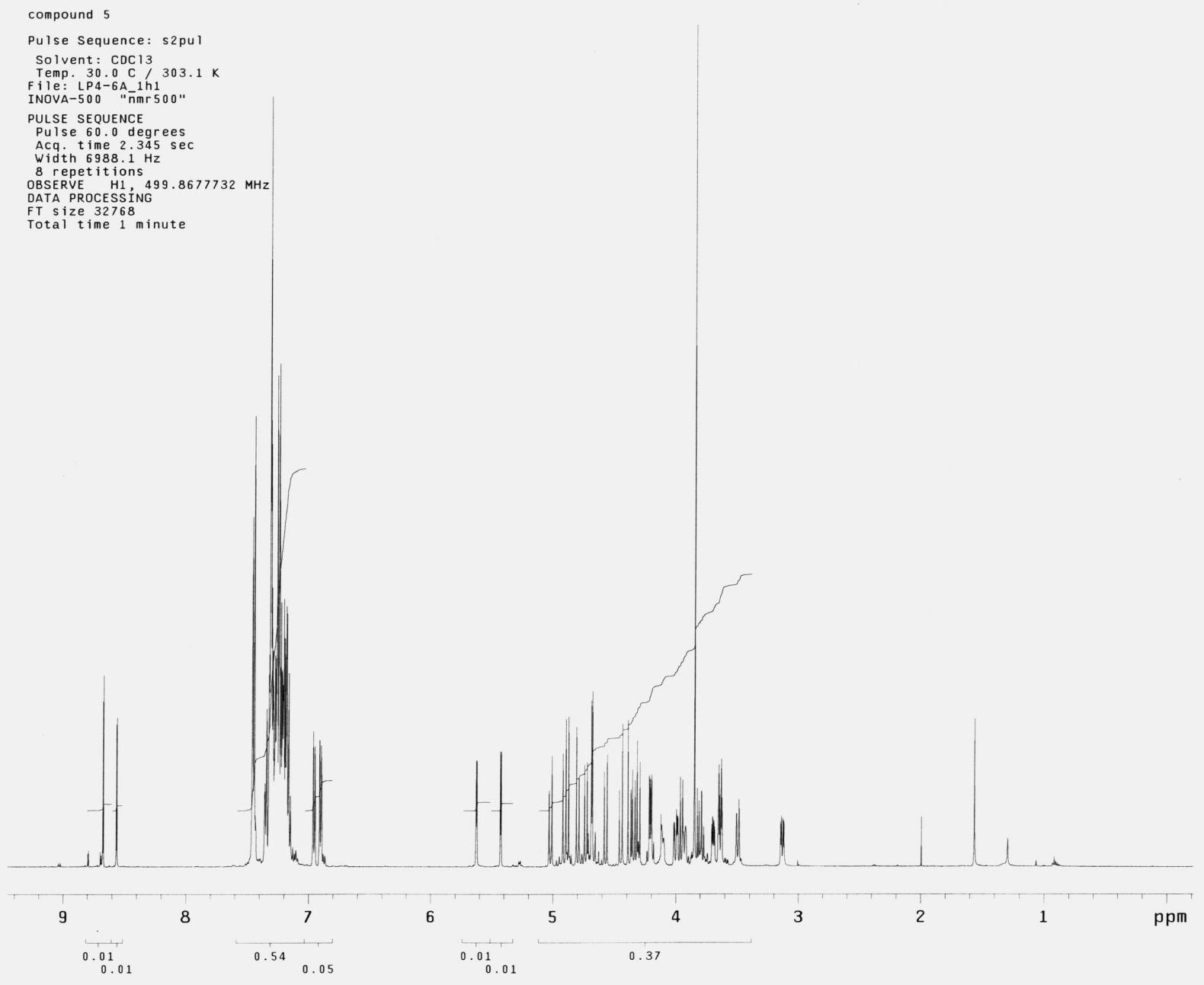

Figure 1: $500 \mathrm{MHz}{ }^{1} \mathrm{H}$ NMR spectra of compound 5. 


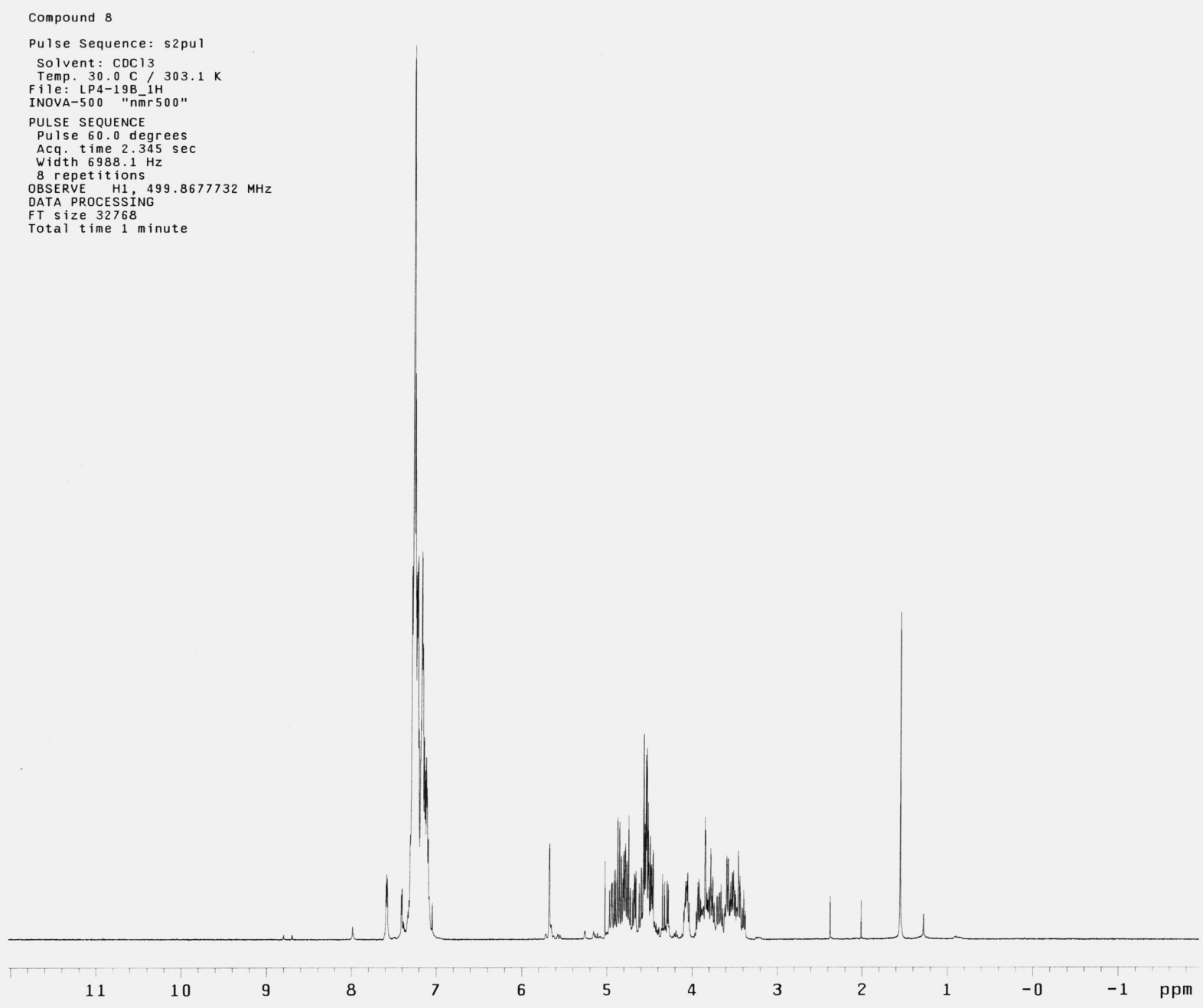

Figure 2: $500 \mathrm{MHz}{ }^{1} \mathrm{H}$ NMR spectra of compound 8 . 


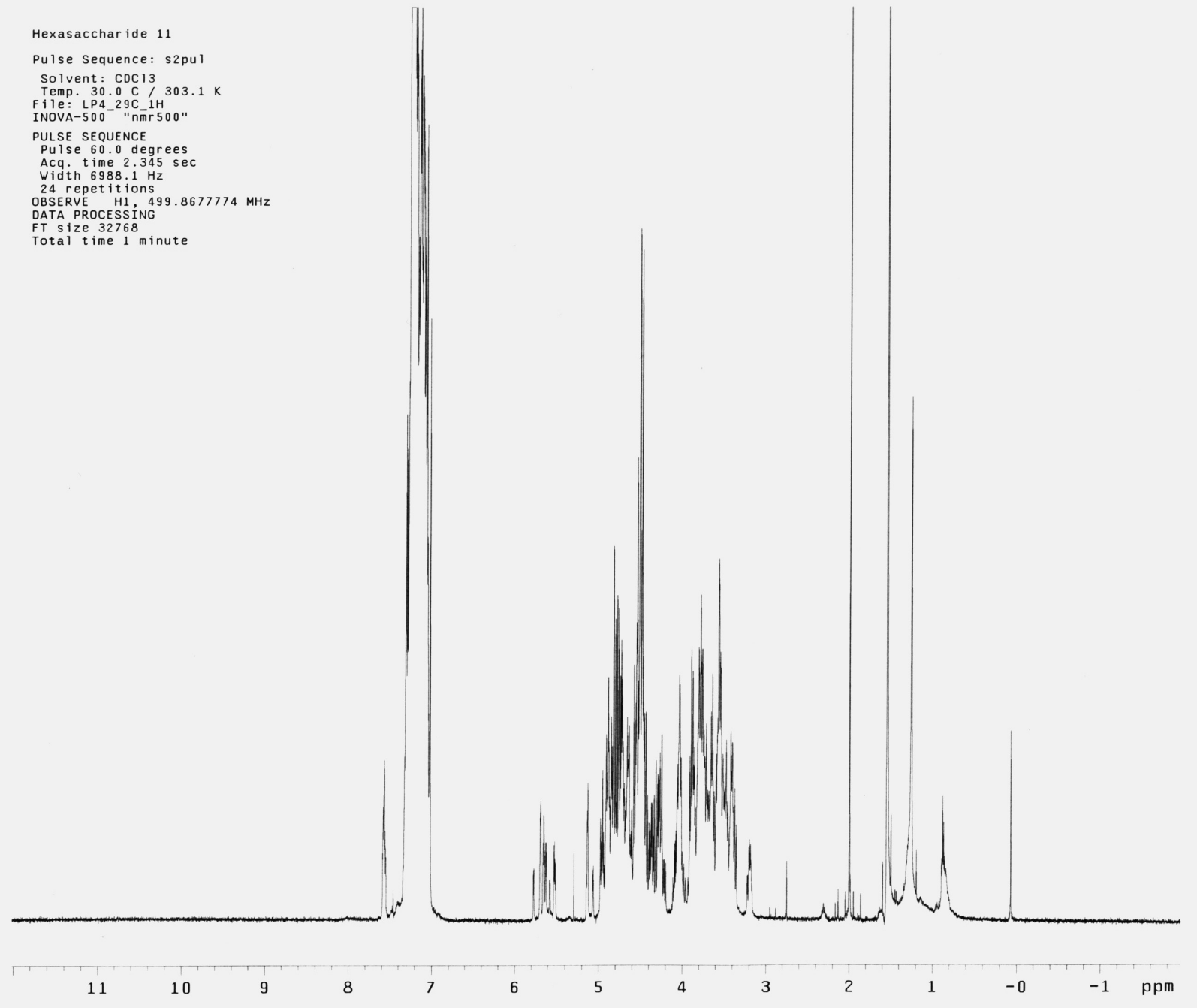

Figure 3: $500 \mathrm{MHz}{ }^{1} \mathrm{H}$ NMR spectra of hexasaccharide 11.

' (a) L. Petersen and K. J. Jensen, J. Org. Chem., 2001, 66, 6268; (b) L. Petersen and K. J. Jensen, J. Chem. Soc. Perkin Trans. 1, 2001, 2175; (c) J. B. Laursen, L. Petersen and K. J. Jensen, Org. Lett., 2001, 3, 687. 